Prof. J. B. S. Haldane suggested that some of the effects, if real, might be due to the transmission of something like a virus, as in the case of the recalcitrant Drosophila. It is a fact that some of the most striking examples claimed by the Russians are in the Solanaceæ, well known as the hosts of viruses sometimes with pseudo-hereditary effects.

While effeets of this kind have been claimed in the past as occurring not infrequently, they may perhaps be considered to be rather rare on the whole. The Russians state that they have used large numbers in their experiments, and it may be that they have come upon an occurrence which has been overlooked in smaller numbers of subjects in work done elsewhere. No amount of appeal to other work can settle the Russian claim; only examination of the Russian material can do that. It is difficult at present to get a clear idea even of the simple facts of the chromosome situation of their plants.

It comes, then, to this. The followers of Lyssenko seem to the formal geneticist to be returning to the methods of the eighteenth century. This was suggested in Nature in $1937^{2}$. But if the methods of breeders then were rule-of-thumb, they gave results which are, some of them, still to be seen. The followers of Lyssenko can retort that the formal geneticists are returning to the methods of the Middle Ages. It was believed then that stars moved in circles. If a star did not seem to be doing this very obviously it was considered proper to assume that the centre of the circle necessarily described in its path must itself be moving in a circle. This epicyclic method of explanation increases the complication of the situation it seeks to explain. This lack of economy is looked upon with disfavour. It affords a tempting refuge for the hard-pressed theoretician. Ptolemy is quoted ${ }^{7}$ as having remarked, perhaps incautiously, that the phenomena could be saved by eccentries and epicycles.

The phenomena do not need saving; they need investigating. It is suggested that now, in view of the recent tragic happenings in the U.S.S.R., many of the Russian varieties of plants and fruittrees may be taken to Great Britain and the United States to be preserved from the invader. It is an irony of history that if this is done it will provide an opportunity for the analysis of the material when no one has time to do it.

\footnotetext{
1 Report of the Conference on Genetics and Selection organized by the Editorial Board of the journal Pod Znamenem Marxisma. Translated for the Society for Cultural Relations, and available for consultation at its offices at 98 Gower Street,'London, W.1. 2 NATURE, 139, 143, 185, 1048 (1937).

" Hammond, J., “Farm Animals", London (1940).

"Muller, H. J., "The New Systematics". Ed. J. S. Huxley, Oxford (1940).

- Haldane, J. B. S., "The Causes of Evolution", London (1932).

- Neilson Jones, W., "Plant Chimaeras and Graft Hybrids", London (1934).

" Sarton, G., "Introduction to the History of Science", 2, Pt. 1., p. 16.
}

\title{
SURFACE CHEMISTRY
}

\author{
By PRoF. W, D. HaRkins \\ UNIVERSITY OF CHICAGO
}

$\mathrm{O}^{\mathrm{N}}$ $\mathrm{NE}$ of the most interesting of the Fiftieth Anniversary Symposia, held at the University of Chicago during September 22-27, was that on two-dimensional systems or surface chemistry. Introductory remarks on the origin and development of this subject at the University were made by Dr. Irving Langmuir, Prof. H. I. Schlesinger, and Prof. W. D. Harkins. [It is interesting to note that this symposium was organized in honour of Prof. Harkins and the twenty-fifth anniversary of the publication of his first paper in the field of surface chemistry.--Editors.] Experimental work on the theory of molecular orientations in surfaces was begun in 1912 on the arrival of Prof. Harkins at the University. The first course of lectures on orientation was presented in the winter quarter of 1913-14. The gist of the theory is contained in a statement from the notes of George L. Clark taken during a lecture which dealt with the more tightly packed films of long-chain organic acids : " $\mathrm{COOH}$ of acid down because both acid and $\mathrm{H}_{2} \mathrm{O}$ associated in polar." This is the earliest recorded statement of the direction of orientation of surface molecules.

In the first problem considered, but not the first to be solved, it was found that the adsorption of polar-nonpolar molecules is much higher at the surface of water than at the interface oil-water, as was predicted by the initial electrical field theory of orientation.

Interest in this problem arose in connexion with work in Karlsruhe in 1909 on Haber's theory of muscular motion, which assumed, as one of several steps, that a change from acid to basic causes a very great lowering of surface tension both in (1) the muscles, and in (2) a benzene-water system, with a strong acid and a strong base as solutes. However, experimental work proved that (2), assumed from work done in Berlin, is incorrect, so it was planned to substitute an organic acid. This gave Prof. Harkins the idea that at the benzene-water interface the hydrocarbon ends of 
the molecules would dissolve in the oil phase and the polar groups in the water, but there was no opportunity to begin a test of this idea until 1912. Later an extensive search through the literature revealed the fact that in 1912 Sir William Hardy expressed the idea that the asymmetrical stray field at the surface of a liquid or a solid causes an orientation of the molecules.

While Hardy gave no evidence in favour of this idea, Harkins found evidence for orientation in Hardy's values for the work of adhesion $\left(W_{A}\right)$ between water and other liquids. Since this evidence was made somewhat uncertain by the inaccuracy in the drop weight method used by Hardy, a considerable amount of experimentation was carried out by Humphery, Brown and Davies, in order to give a high degree of accuracy to the method. This was the more necessary on account of the fact that the internal (surface) energy $(E)$ is much more intimately related to the molecular orientation than is the free surface energy $(F)$, and the determination of $E$ involves the entropy, that is, the slope $\left(\partial_{\gamma} / \partial T\right)_{p_{1} \sigma}$, where $\sigma$ equals molecular area.

Even at the present time one of the best lines of evidence for the orientation of molecules in interfaces is given by the values of $W_{A}$, and especially of $E_{A}$, at the liquid-liquid, and at the solid-liquid interface. For example, the energy $E_{C}$ in erg.cm.-2 required to pull $n$-octane apart is 100 , while for $n$-octyl alcohol it is only 101, showing that the polar group has almost no effect, since the molecules orient so that only the non-polar hydrocarbon groups are pulled apart. To pull $n$-octane from water requires an energy of 107 , but to pull $n$-octyl alcohol from water a much higher energy is needed (165), since the polar group must be separated from the water.

In recent work with Dr. G. E. Boyd the relative energies of immersion $\left(E_{I}\right)$ of $\mathrm{TiO}_{2}$ (anatase) are found to be: water, 1.00 ; butyric acid, 0.77 ; ethyl acetate, 0.69 ; butyl alcohol, 0.67 ; nitrobenzene, 0.55 ; carbon tetrachloride, 0.46 ; benzene, $0 \cdot 28$; iso-octane, $0 \cdot 20$, and these ratios are practically the same for the solids, which give the following vatues of the total energy of adhesion $\left(E_{A}\right)$ in erg.cm.-2 $: \mathrm{BaSO}_{4}, 490 ; \mathrm{TiO}_{2}, 520 ; \mathrm{SiO}_{2}, 600$; $\mathrm{ZrO}_{2}, 600 ; \mathrm{SnO}_{2}, 680$, and $\mathrm{ZrSiO}_{4}, 850$, graphite 265. Graphite, however, exhibits abnormal values of the ratio, namely, 0.73 for $\mathrm{CCl}_{4}$ and 0.85 for benzene. All the crystalline solids (ex́cept graphite) are found to be much more polar than water and the polar groups are turned towards the solid.

Values obtained by the adsorption of four vapours by $H$. K. Livingston show that all give the same area for any particular solid, provided the areas per molecule in the completed first mono- layer are taken as: nitrogen, 15.4 ; water, $10 \cdot 6$; propyl alcohol, $20 \cdot 0$; and $n$-heptane, $55.0 \mathrm{~A}^{2}$. The last value shows that in the completed monolayer n-hydrocarbon molecules lie flat on the surface.

In his paper on the properties of solid surfaces, Dr. Boyd gave examples of the free energy of adhesion between a solid and various liquids. With $\mathrm{TiO}_{2}$ the values are: water, 380 ; propyl alcohol, 129 ; and $n$-heptane, 78 . The corresponding values of $\gamma_{S} \gamma_{S V_{0}}$ (energy of immersion in the saturated vapour) are 240,82 and $38 \mathrm{erg} . \mathrm{cm}^{-2}$ for the clean surface of anatase. The values were calculated from the vapour adsorption isotherms obtained by Livingston and the proper Gibbs's adsorption equation.

Dr. Fritz London, of Duke University, considered the centres of van der Waals' attraction. A special type of long-range attraction is assumed to act between large molecules where the virtual electron oscillators are of appreciable extension.

If a molecule possesses considerable electrical anisotropy in its parts (as in the chemical bonds), it is no longer permissible to use the dispersion force as a central force, but rather specific formulæ must be employed. For example, the interaction energy between two anisotropic force centres (bond ellipsoids) is :

$$
\begin{array}{r}
E_{\mathrm{I}, \mathrm{II}}=-\frac{1}{R^{6}}\left[A-B-B^{\prime}+C\left(\sin \delta \sin \delta^{\prime} \cos \varphi-\right.\right. \\
\left.2 \cos \delta \cos \delta^{\prime}\right)^{2}+3(B-C) \cos ^{2} \delta+ \\
\left.3\left(B^{\prime}-C\right) \cos \delta^{\prime}+B+B^{\prime}+4 C\right],
\end{array}
$$

where $R$ is the distance between the centres I and II, $\alpha_{\| 1} \alpha_{1}$ and $\alpha_{\| \mid}^{\prime}, \alpha_{1}^{\prime}$, the components of their polarizabilities, respectively, $\bar{v}_{\| \prime}, \bar{v}_{\perp}$ and $\bar{v}_{\| 1}, \bar{v}_{\perp}$ the corresponding frequencies, $\delta, \delta^{\prime}$, the slopes of the two bond directions with respect to the line joining the centres, and $\varphi$ the angle between the projections of the two bond axes into a plane perpendicular to the joining line.

In the special case where the interaction energy between large oscillators is desired for distances shorter than the spacial extension of the interacting virtual charge distributions of the molecules, it was found desirable to represent each oscillator by several distinct poles, 'monopoles', of different sign, suitably located in the molecule. As a consequence of the quantum mechanical treatment of the problem in these terms a new formula for the energy of interaction is reached:

$$
E^{(2)}=\frac{\varepsilon^{2} \varepsilon^{\prime 2}}{h\left(\nu+\nu^{\prime}\right)}\left[\frac{1}{R_{++}}+\frac{1}{R_{--}}-\frac{1}{R_{+-}}-\frac{1}{R_{-+}}\right]^{2}
$$

where $R_{++}, R_{--}, R_{+-}, R_{-+}$symbolize the distance of separation of the respective monopoles; $\varepsilon, \varepsilon^{\prime}$ give the magnitude of the high-frequency dipoles.

The theory should be useful for systems containing conjugated double bonds, of which rubber is an example, and for large biological molecules. 
Prof. J. G. Kirkwood, of Cornell University, discussed the theory of the transition expanded to the intermediate liquid phase. This transition is considered as an order-disorder transformation in axial orientation of the molecules which constitute the film. By postulating a free energy barrier of appropriate form, which hinders the relative axial rotation of neighbouring molecules, Kirkwood is able to demonstrate a phase change of the second order in a model film of cylindrical molecules of elliptical cross-section vertically oriented on the subphase. By means of the statistical mechanical theory of co-operative phenomena the magnitude of the discontinuities in the heat capacity, the coefficients of thermal expansion and compressibility attending the transition, have been calculated. These agree well with the experimental values of Harkins, Young and Boyd.

Surface entropy of pure liquids was dealt with in a paper by Mr. Henry Eyring of Princeton University.

A relation between free surface energy $\left(A_{S}\right)$ and the Eötiös equation is :

$$
A_{S}=E_{S}-T S_{S}=N^{1 / 3} V^{2 / 3}=4 \cdot 4\left(T_{C}-T-6\right)
$$

The entropy, 4.4 Cal. mole ${ }^{-1}$ deg..$^{-1}$ arises from expansion of the surface region, the free energy of which can be estimated by the partition function of normal liquids (Walter and Eyring). The final equation is $\gamma=\left(\rho_{L}-\rho_{V}\right)(a-b T)$ where $a / b \sim T_{C}$.

Remarkable electron microscopophotographs of gas carbon, bacteria and the coatings of insects were presented by Prof. E. F. Burton, of the University of Toronto, the pioneer in this field in. America.

Dr. George H. A. Clowes, research director of Eli Lilly and Co., described the interactions of biologically significant substanees.

From extremely extensive data it is shown that many carcinogenic polycyclic hydrocarbons are highly soluble in sterol monolayers (10-methyl-1-2 benzanthracene in cholesterol), in which case the logarithm of the mol fraction of the hydrocarbon varies linearly with film pressure $(\pi)$. A complex of one molecule of hydrocarbon to two of sterol forms with a free energy of 2.5 kilocalories or less. Increase of pressure on a two-dimensional solution may force the hydrocarbon into colloidal solution in the subphase. Such material penetrates the film at lower pressures. The relations to biology were discussed.

The intrinsic viscosities and the reciprocals of the frictional ratio of fifteen globular proteins were compared by Prof. Henry B. Bull and Mr. J. A. Cooper, of Northwestern University, and an empirical, linear relation found to exist between them. This empirical relation was used to estimate the average volume hydration, which was found to be 0.283 c.c. per c.c. of dry protein, with a standard deviation of the mean of 0.042 c.c.

A test applied to several theoretical equations in the literature relating viscosity and asymmetry of suspended particles is based upon the empirical linear relation between viscosities and reciprocals of the frictional ratios of the protein molecules. None of the theoretical viscosity equations are valid when applied to protein solutions.

Increase of surface tension $(\gamma)$ by highly polar organic compounds was the subject of a paper by Prof. Ernst A. Hauser, of the Massachusetts Institute of Technology, and Mr. Adrian J. Grossman.

The increments of surface tension produced in benzene by a 0.5 mole fraction at $40^{\circ}$ of dinitrobenzenes are: ortho $1 \cdot 75(6.05)$, meta $1 \cdot 3(3 \cdot 81)$, para $1.2(0.32)$, in dyne $\mathrm{cm} .^{-1}$, where the values in parentheses are the dipole moments. Thus the increments in $\gamma$ are related more intimately to the high bond moment $(3.9)$ of the nitro group than to the molecular moment. Frequent changes in the slope $(\delta \gamma / \delta C)_{T, p}$ were found, and a deep depression of 1.2 dyne $\mathrm{cm} .^{-2}$ was obtained with 1-nitronaphthalene.

Mr. Eugene Guth, of the University of Notre Dame, considered stress and elasticity in rubber. The theory presented on the basis of an ideal model of incompressible rubber considers an irregu. lar network of flexible long-chain molecules, and predicts the characteristic S-shaped stress-strain curves found in experiments on an unaccelerated pure gum compound. In the analytical expression for the theoretical curves, the Langevin function of the theory of paramagnetism, or the Debye theory of dipole molecules, enters and describes the orientation of the long molecules due to the applied stress.

It is found :

(1) That the stress is due largely to the kinetic energy (entropy) of the molecules and not to the intermolecular forces, since the latter are found to contribute only about 20 per cent of the stress.

(2) As predicted by the theory the stress at constant length is proportional to the absolute temperature.

(3) The slope of the stress-temperature curves is negative up to 10 per cent extension and is positive above this, due to thermal expansion.

(4) Since the heat developed in adiabatic stretching is proportional to the temperature coefficient of the stress (Kelvin-Joule), heat is absorbed for $0-10$ per cent extension, and is given off for greater extensions.

A new superliquid phase was described by Prof. W. D. Harkins, Copeland and G. E. Boyd.

At certain temperatures it is found that the condensed liquid $\left(L_{2}\right)$ phase of a long-chain alcohol 
on increase of pressure transforms by a first order change into a new $L S$ phase, which has the compressibility of the solid phase, but exhibits a greatly lowered viscosity compared with that of the liquid from which it is formed by compression and the addition of heat. The internal energy increase and the increase of entropy when this phase expands are excessively large. In the transformation of the $L_{2}$ to the solid $S$ phase there is no latent heat, so the change is second order.

Mr. Eyring considered the viscosity of monolayers from the theory of ahsolute reaction rates.

A decrease in viscosity with increase in pressure indicates that the activated state occupies a smaller area than the initial state, and vice versa. Similarly a decrease in viscosity with temperature indicates that the activated state has a greater energy than the initial state; and increase in viscosity with temperature that the activated has less energy than the normal state.

A thermodynamical theory of the spreading of liquids on surfaces was presented by Prof. Harkins.

(1) A duplex $(D)$ or thick film may spread if its spreading involves a decrease of free energy. On water a $D$ film is always unstable, since there is a decrease of free energy when it transforms into a monolayer $(M)$ and a lens.

(2) Water and all oils spread on mercury, and all liquid oils on water to give monolayers, but if a duplex film cannot spread the pressure $(\pi)$ of the monolayer may be small.

(3) $\pi_{e}=S_{b^{\prime} / a}-S_{b^{\prime} / a^{\prime}}$ or the semi-initial minus the final spreading coefficient. Since $S_{b^{\prime} / a}$ is always negative, $\pi_{e}$, the equilibrium pressure, is always greater than the semi-initial spreading coefficient, and almost always greater than $S_{b \mid a s}$ the initial coefficient for the spreading of the dry oil $(b)$ on clean water $(a)$ as a duplex film.

(4) Water does not spread as a duplex film on any oil.

(5) It always takes less work to pull any oil from its first complete monolayer on water than to pull the oil apart.

(6) While lower hydrocarbons give duplex film spreading on water, higher hydrocarbons and some polar oils (as methylene iodide) do not, but form monolayers only.

Dr. Irving Langmuir, of the General Electric Co., discussed the permeability of monolayers.

Copper gauze supporting a layer of $\mathrm{CaCl}_{2} \cdot a \mathrm{H}_{2} \mathrm{O}$, mounted at a distance $b$ above the surface of water in a film trough is used to measure the rate of evaporation. At equilibrium, the rate at which water escapes is determined by the resistance of the moisture-saturated air in the space between the liquid surface and the adsorbent. This resistance to diffusion, $\omega$, is given by $\omega=A w t / M=b / D$, where $A$ is the surface area evaporating, $w$ the grams of water per unit volume of saturated air, $t$ the time in seconds, and $M$ the mass evaporated ; $D$ is the diffusion coefficient. A monomolecular film of an acid, alcohol, cholesterol or ergosterol placed on a clean water surface gives an increase in $\omega$, but proteins do not. A logarithmic relation is found between $\omega$ and the film pressure. The permeability exhibits extreme sensitivity to impurities. Langmuir considers an impermeable film to be tightly packed at some definite height in the film.

\section{NEWS AND VIEWS}

\section{In Defence of Liberty}

LAST week, on the outbreak of war between the United States and the Totalitarian Powers, the King sent a message to President Roosevelt, which concluded with the words: "We share your inflexible determination and your confidence that, with God's help, the powers of darkness will be overcome and the four freedoms established throughout a world purged of tyranny." President Roosevelt, in his reply, paid a tribute to the courage of the British people during the past two years, and said: "The forces which have plunged the world in war, however strong, cannot prevail against the indomitable strength of free peoples fighting in a just cause."

This was also the text of President Roosevelt's stirring radio address to the American peoples on December 15, the one hundred and fiftieth anniversary of the adoption by Congress of the Bill of Rights The basic principles of the freedom of man embodied in the Bill have been accepted by all the republics of the Western hemisphere, and indeed by some four fifths of the peoples of the world. The present struggle is nothing less than an attempt on the part of the Totalitarian Powers to overthrow all the results that have flowed from the gradual growth of the liberty of the individual. It is an attempt to impose once more on mankind the tyranny and despotic rule of the Middle Ages, from which we have been set free by the courage and sacrifice of our ancestors. The present generation of Americans, President Roosevelt said, are as determined to preserve liberty as their ancestors were to win it, and he pledged Americans not to lay down the arms they have now taken up until liberty is once more secure in the world. 